\title{
The Modified Theory of Central-Force Motion
}

\author{
${ }^{1}$ Edison A. Enaibe,(Ph.D.) $)^{2}$ Emmanuel E. Enukpere, (M.Sc.) and \\ ${ }^{3}$ John O. A. Idiodi, (Prof.) \\ 1. Department of Physics, Federal University of Petroleum Resources, Effurun, Nigeria. \\ 2. Department of Physics, College of Education, Warri, Nigeria. \\ 3. Department of Physics, University of Benin P.M.B. 1154, Benin City, Edo State, Nigeria.
}

\begin{abstract}
Everybody is in the state of constant vibration, since vibration is the cause of the entire universe. Consequent upon this, a body under the influence of attractive force that is central in character may also vibrate up and down about its own equilibrium axis as it undergoes a central-force motion. The up and down vertical oscillation would cause the body to have another independent generalized coordinates in addition to the one of rotational coordinates in the elliptical plane. In this work, we examine the mechanics of a central-force motion when the effect of vertical oscillation is added. The differential orbit equation of the path taken by the body varies maximally from those of the usual central-force field. The path velocity of the body converges to the critical speed in the absence of the tangential oscillating phase.
\end{abstract}

Keywords: Elliptical plane, upper and lower radial orbital oscillating angles, upper and lower vertical oscillating angles, vertical oscillation, critical velocity, drag oscillating force.

\section{Introduction}

A central force is a conservative force. It is a force directed always toward or away from a fixed center $O$ and whose magnitude is a function only of the distance from $O$ [1]. In spherical coordinates, with $O$ as origin, a central force is given by $F=f(r) \hat{r}$. Physically, such a force represents an attraction if $(f(r) \prec 0)$ and repulsion if $(f(r) \succ 0)$, from a fixed point located at the origin $r=0$.

Examples of attractive central forces are the gravitational force acting on a planet due to the sun. Nuclear forces binding electrons to an atom undoubtedly have a central character. The force between a proton or an alpha particle and another nucleus is a repulsive central force.

The relevance of the Central - force motion in the macroscopic and microscopic frames warrants a detailed study of the theoretical mechanics associated with it.

So far, researchers have only considered central - force motion, as motion only in the translational and rotational plane with coordinates $(r, \theta)$, for example, see Keplerian orbits [2, 3]. However, the theoretical knowledge advanced by these researchers in line with this type of motion is scientifically restricted as several possibilities are equally applicable.

There exist four standard formulations of classical mechanics: (i) Isaac Newton's formulation - Newtonian mechanics (ii) Lagrange's formulation - Lagrangian mechanics (iii) Hamilton's formulation - Hamiltonian mechanics (iv) De Alambert's formulation - De Alambertian mechanics. All these formulations are utilized in the theory of mechanics where applicable.

Some of the conditions satisfied by a body undergoing a Central - force motion is as follows: (i) the motion of the body can be translational and rotational in the elliptical plane with polar coordinates $(r, \theta)$, (ii) the body can be rotating and revolving about its own axis in the elliptical plane $(r, \theta)$, (iii) the body can be translating and rotating in the elliptical plane $(r, \theta)$, at the same time, oscillating up and down about its own axis (iv) the body can be translating and rotating in the elliptical plane $(r, \theta)$, at the same time, oscillating up and down but not below its axis of rotation (v) the combination of any of these conditions form another class of a Central - force motion.

In order to make the mechanics of a Central - force motion sufficiently meaningful, we have in this work extended the theory which has only been that of translational and rotational in the elliptical plane with polar coordinates $(r, \theta)$, by including vertical spin oscillation.

Under this circumstance, we shall be contending with a total of 6 - generalized coordinates or degrees of freedom; 2 from the translational and rotational motion in the elliptical plane $(r, \theta), 2$ from the orbital spin oscillations $(\beta, \alpha)$ and 2 from the tangential spin oscillations $(\mu, \phi)$. Consequently, these parameters form the basis of our classical theory of 6-dimensional motion. 
The number of independent ways in which a mechanical system can move without violating any constraints which may be imposed is called the number of degrees of freedom of the system. The number of degrees of freedom is the number of quantities which must be specified in order to determine the velocities of all particles in the system for any motion which does not violate the constraints [4].

There is a single source producing the force that depends only on distance in the theory of central-force motion and the force law is symmetric [5]. If this is the case, then, there can be no torques present in the system as there would have to be a preferred axis about which the torques acts.

In this work, we are solving the problem of oscillating central force motion in a resistive non-symmetric system. That is, the upward displacement is not equal to the downward displacement in the tangential spin oscillating phase. Consequently, the radii distances from the central point are not equal. This however, causes torques thereby making the system under study non-spherically symmetric.

Meanwhile, I hereby request the permission of the reader to excuse the lack of intensive references to the current literature. I don't know of other current authors who have studied these questions before now. I believe this is the first time this study is under investigation.

This paper is outlined as follows. Section 1, illustrates the basic concept of the work under study. The mathematical theory is presented in section 2 . While in section 3 , we present the analytical discussion of the results obtained. The conclusion of this work is shown in section 4 and this is immediately followed by appendix and list of references.

\section{Research methodology}

The analytical geometry of the work is first figuratively represented. This provides the pictorial understanding of the work under investigation and the possibility of specifying the required generalized coordinates. Thereafter, simple rule of trigonometry is used to define the vector quantities which we need for the evaluation of the physical quantities. Finally, differential techniques in combination with the theory of classical mechanics are utilized in the discussion of the problem of the modified theory of central-force motion.

\subsection{Evaluation of the velocity and acceleration}

\section{Mathematical theory}

We have elaborately shown in (A. 6) in the appendix that the position vector $\vec{r}$ of a body whose motion is translational and rotational in a plane polar orbit as well as oscillating about a given equilibrium position in a central-force motion is given by the equation

$$
\begin{aligned}
& \vec{r}=r \hat{r}=r \hat{r}(\theta, \beta, \mu, \alpha, \phi)(2.1) \\
& v=\frac{d \vec{r}}{d t}=\frac{d r}{d t} \hat{r}+r\left(\frac{d \hat{r}}{d \theta} \frac{d \theta}{d t}+\frac{d \hat{r}}{d \beta} \frac{d \beta}{d t}+\frac{d \hat{r}}{d \mu} \frac{d \mu}{d t}+\frac{d \hat{r}}{d \alpha} \frac{d \alpha}{d t}+\frac{d \hat{r}}{d \phi} \frac{d \phi}{d t}\right) \\
& v=\dot{r} \hat{r}+r \dot{\theta} \hat{\theta}+r \dot{\beta} \hat{\beta}+r \dot{\mu} \hat{\mu}+r \dot{\alpha} \hat{\alpha}+r \dot{\phi} \hat{\phi} \\
& a=\frac{d^{2} \vec{r}}{d t^{2}}=\frac{d v}{d t}=\ddot{r} \hat{r}+\dot{r}\left(\frac{d \hat{r}}{d \theta} \frac{d \theta}{d t}+\frac{d \hat{r}}{d \beta} \frac{d \beta}{d t}+\frac{d \hat{r}}{d \mu} \frac{d \mu}{d t}+\frac{d \hat{r}}{d \alpha} \frac{d \alpha}{d t}+\frac{d \hat{r}}{d \phi} \frac{d \phi}{d t}\right) \\
& +\dot{r} \dot{\theta} \hat{\theta}+r \ddot{\theta} \hat{\theta}+r \dot{\theta}^{2}\left(\frac{d \hat{\theta}}{d \theta}\right)+\dot{r} \dot{\beta} \hat{\beta}+r \ddot{\beta} \hat{\beta}+r \dot{\beta}^{2}\left(\frac{d \hat{\beta}}{d \beta}\right)+\dot{r} \dot{\mu} \hat{\mu}+r \ddot{\mu} \hat{\mu}+r \dot{\mu}^{2}\left(\frac{d \hat{\mu}}{d \mu}\right) \\
& +\dot{r} \dot{\alpha} \hat{\alpha}+r \ddot{\alpha} \hat{\alpha}+r \dot{\alpha}^{2}\left(\frac{d \hat{\alpha}}{d \alpha}\right)+\dot{r} \dot{\phi} \hat{\phi}+r \ddot{\phi} \hat{\phi}+r \dot{\phi}^{2}\left(\frac{d \hat{\phi}}{d \phi}\right) \\
& a=\left(\ddot{r}-r \dot{\theta}^{2}\right) \hat{r}+(r \ddot{\theta}+2 \dot{r} \dot{\theta}) \hat{\theta}+\left(r \ddot{\beta}+2 \dot{r} \dot{\beta}-r \dot{\beta}^{2} \tan \beta\right) \hat{\beta}+\left(r \ddot{\mu}+2 \dot{r} \dot{\mu}-r \dot{\mu}^{2} \tan \mu-2 r \dot{\mu}^{2} \cot \mu\right) \hat{\mu} \\
& +\left(r \ddot{\alpha}+2 \dot{r} \dot{\alpha}-r \dot{\alpha}^{2} \tan \alpha\right) \hat{\alpha}+\left(r \ddot{\phi}+2 \dot{r} \dot{\phi}-r \dot{\phi}^{2} \tan \phi-2 r \dot{\phi}^{2} \cot \phi\right) \hat{\phi}(2.5)
\end{aligned}
$$

where the symbols appearing in (2.1) - (2.5) have been clearly defined in the appendix.

However, let us disengage the acceleration equation in $(2.5)$ with the view that the $5^{\text {th }}$ and the $8^{\text {th }}$ terms have the elements of angular momentum as a constant of the motion and the radial orbital oscillating phases. Thus

$a=\left(\ddot{r}-r \dot{\theta}^{2}\right) \hat{r}+(r \ddot{\theta}+2 \dot{r} \dot{\theta}) \hat{\theta}+(r \ddot{\beta}+2 \dot{r} \dot{\beta}) \hat{\beta}-\left(r \dot{\beta}^{2} \tan \beta\right) \hat{\beta}+\left(r \ddot{\mu}+2 \dot{r} \dot{\mu}-r \dot{\mu}^{2} \tan \mu-2 r \dot{\mu}^{2} \cot \mu\right) \hat{\mu}$ 
$+(r \ddot{\alpha}+2 \dot{r} \dot{\alpha}) \hat{\alpha}-\left(r \dot{\alpha}^{2} \tan \alpha\right) \hat{\alpha}+\left(r \ddot{\phi}+2 \dot{r} \dot{\phi}-r \dot{\phi}^{2} \tan \phi-2 r \dot{\phi}^{2} \cot \phi\right) \hat{\phi}(2.6)$

Equation (2.6) is now the required new acceleration equation which governs the motion of a body undergoing a central-force motion when the effect of drag oscillating force is added.

\subsection{Evaluation of the central - force field}

In classical mechanics, a central force is a force whose magnitude only depends on the distance $r$, of the body from the origin and is directed along the line joining them [5]. Thus, from the analytical geometry of the central-force motion shown in fig. A. 1, in the appendix, permits us to write in terms of vector algebra

$$
F(r)=f(\|r\|)(\hat{r} ; \hat{\beta}, \hat{\alpha})=f(\|r\|) \hat{r}+f(\|r\|) \hat{\beta}+f(\|r\|) \hat{\alpha}=m a(2.7)
$$

where $F$ is a vector valued force function, $f$ is a scalar valued force function, $r$ is the position vector, $\|r\|$ is its length, and $\hat{r}=r /\|r\|$, is the corresponding unit vector.

We can convert (2.6) to force by simply multiplying it by the mass $m$ of the body and equate the resulting expression to (2.7). Note that we are utilizing the radial orbital oscillating phase in (2.6), which is acting in the directions of $\hat{\beta}$ and $\hat{\alpha}$ in our calculation. Once this is done, we obtain the following sets of canonical equations of motion.

$$
\begin{aligned}
& f(r)=m\left\{\left(\ddot{r}-r \dot{\theta}^{2}\right)-\left(r \dot{\beta}^{2} \tan \beta+r \dot{\alpha}^{2} \tan \alpha\right)\right\} \\
& m(r \ddot{\theta}+2 \dot{r} \dot{\theta})=0(2.9) \\
& m(r \ddot{\mu}+2 \dot{r} \dot{\mu})=0(2.10) \\
& m(r \ddot{\beta}+2 \dot{r} \dot{\beta})=0 \\
& m(r \ddot{\alpha}+2 \dot{r} \dot{\alpha})=0(2.12) \\
& m(r \ddot{\phi}+2 \dot{r} \dot{\phi})=0
\end{aligned}
$$

Equation (2.8) is the required new central-force field which we have developed in this study. It is 4-dimensional and it governs the central-force motion of a body when the effect of drag oscillating force is added. In this equation, $r \dot{\theta}^{2}$ is the centripetal acceleration arising from the $\theta$ direction and $2 \dot{r} \dot{\theta}$ is often referred to as the Coriolis acceleration.

The sets of canonical equation (2.9) - (2.13) determines the angular momentum which are the constants of the motion in the directions of increasing coordinates, $\theta, \beta, \mu, \alpha$ and $\phi$.

\subsection{Relationship between the radial velocity and the tangential oscillating angles}

In consideration of the vertical oscillating motion in the direction of $\mu$ in (2.6), we have that for $m \neq 0$

$$
\begin{aligned}
& \left(r \ddot{\mu}+2 \dot{r} \dot{\mu}-r \dot{\mu}^{2} \tan \mu-2 r \dot{\mu}^{2} \cot \mu\right)=0 \\
& \dot{\mu}=\frac{2 \dot{r} \pm \sqrt{4 \dot{r}^{2}+4 r^{2} \ddot{\mu}(\tan \mu+2 \cot \mu)}}{2 r(\tan \mu+2 \cot \mu)}(2.15) \\
& \dot{\mu}=\frac{2 \dot{r} \pm 2 \dot{r} \sqrt{\left(1+\frac{r^{2}}{\dot{r}^{2}} \ddot{\mu}(\tan \mu+2 \cot \mu)\right)}}{2 r(\tan \mu+2 \cot \mu)}
\end{aligned}
$$

The discriminate of (2.16) is zero provided

$$
\begin{aligned}
& \dot{r}^{2}=-r^{2} \ddot{\mu}(\tan \mu+2 \cot \mu)(2.17) \\
& |\dot{r}|=r \sqrt{\ddot{\mu}(\tan \mu+2 \cot \mu)}(2.18)
\end{aligned}
$$

Similarly, by following the same algebraic procedure, we obtain the radialvelocity acting in the direction of the verticaloscillating motion $\phi$ as

$$
|\dot{r}|=r \sqrt{\ddot{\phi}(\tan \phi+2 \cot \phi)}(2.19)
$$

Thus the radial velocity $\dot{r}$ is directly proportional to the radius vector and to the square root of the vertical oscillating angles. Therefore, the radial velocity decreases as the vertical spin oscillating angles is increased. 


\subsection{Evaluation of the Differential orbit equation (DOE) for the central- force field}

The motion of a body undergoing a central force motion would have to follow a defined trajectory as it orbits the central point (focus). Now, equation (2.9) can be compactly written as

$$
\begin{aligned}
& m(r \ddot{\theta}+2 \dot{r} \dot{\theta})=0 \quad ; \quad \frac{m}{r}\left(r^{2} \ddot{\theta}+2 r \dot{r} \dot{\theta}\right)=0 \quad ; \quad\left(r^{2} \ddot{\theta}+2 r \dot{r} \dot{\theta}\right)=0 \quad ; \quad(m / r \neq 0)(2.20) \\
& \frac{d}{d t}\left(r^{2} \dot{\theta}\right)=0 \quad ; \quad\left(r^{2} \dot{\theta}\right)=h \quad ; \quad \dot{\theta}=\frac{h}{r^{2}}
\end{aligned}
$$

Following the same method as that of (2.9) which leads to (2.20) and (2.21), we get respectively for the other equations (2.10) - (2.13), that

$$
\begin{aligned}
& \frac{d}{d t}\left(r^{2} \dot{\mu}\right)=0 \quad ; \quad\left(r^{2} \dot{\mu}\right)=h_{1} \quad ; \quad \dot{\mu}=\frac{h_{1}}{r^{2}} \\
& \frac{d}{d t}\left(r^{2} \dot{\beta}\right)=0 \quad ; \quad\left(r^{2} \dot{\beta}\right)=h_{1} \quad ; \quad \dot{\beta}=\frac{h_{1}}{r^{2}} \\
& \frac{d}{d t}\left(r^{2} \dot{\alpha}\right)=0 \quad ; \quad\left(r^{2} \dot{\alpha}\right)=h_{2} \quad ; \quad \dot{\alpha}=\frac{h_{2}}{r^{2}} \\
& \frac{d}{d t}\left(r^{2} \dot{\phi}\right)=0 \quad ; \quad\left(r^{2} \dot{\phi}\right)=h_{2} \quad ; \quad \dot{\phi}=\frac{h_{2}}{r^{2}} \\
& r=\frac{1}{u} \quad ; \quad r u=1 ; \quad \frac{d r}{d u}=-\frac{1}{u^{2}} \\
& \dot{r}=\frac{d r}{d t}=\frac{d r}{d \theta} \frac{d \theta}{d t}=\dot{\theta} \frac{d r}{d \theta}=\frac{h}{r^{2}} \frac{d r}{d \theta} \\
& \dot{r}=\frac{h}{r^{2}}\left(\frac{d r}{d u} \frac{d u}{d \theta}\right)=\frac{h}{r^{2}}\left(-\frac{1}{u^{2}} \frac{d u}{d \theta}\right)=-\frac{h}{u^{2} r^{2}}\left(\frac{d u}{d \theta}\right)=-h\left(\frac{d u}{d \theta}\right) \\
& \ddot{r}=\frac{d \dot{r}}{d t}=\frac{d}{d t}\left(-h \frac{d u}{d \theta}\right)=-h \frac{d}{d t}\left(\frac{d u}{d \theta}\right)=-h \frac{d}{d \theta}\left(\frac{d u}{d t}\right) \\
& \ddot{r}=-h \frac{d}{d \theta}\left(\frac{d u}{d \theta} \frac{d \theta}{d t}\right)=-h \dot{\theta}\left(\frac{d^{2} u}{d \theta^{2}}\right)=-h\left(\frac{h}{u^{2}}\right)\left(\frac{d^{2} u}{d \theta^{2}}\right)=-h^{2} u^{2}\left(\frac{d^{2} u}{d \theta^{2}}\right)
\end{aligned}
$$

By utilizing (2.21), (2.23), (2.24) and (2.30) in the new central force field equation given by (2.8), we get

$$
m\left\{-h^{2} u^{2} \frac{d^{2} u}{d \theta^{2}}-r\left(\frac{h^{2}}{r^{4}}\right)-r\left(\frac{h_{1}^{2}}{r^{4}}\right) \tan \beta-r\left(\frac{h_{2}^{2}}{r^{4}}\right) \tan \alpha\right\}=F(r ; \beta, \alpha)=F(r)
$$

It is assumed in this study that $P$ and $Q$ are very small displacements from the equilibrium axis of rotation. As a result, we write $h_{1} \approx h_{2} \approx h$, and if we make the substitution $r=1 / u$ in (2.31), then

$$
\begin{aligned}
& m\left\{-h^{2} u^{2} \frac{d^{2} u}{d \theta^{2}}-h^{2} u^{3}-h^{2} u^{3} \tan \beta-h^{2} u^{3} \tan \alpha\right\}=F(1 / u)(2.32) \\
& \left\{-\frac{d^{2} u}{d \theta^{2}}-u-u \tan \beta-u \tan \alpha\right\}=-\frac{F(1 / u)}{m h^{2} u^{2}}(2.33) \\
& \left\{\frac{d^{2} u}{d \theta^{2}}+(\tan \beta+\tan \alpha) u+u\right\}=-\frac{F(1 / u)}{m h^{2} u^{2}}(2.34)
\end{aligned}
$$

Equation (2.34) is the required differential orbit equation for oscillating central-force motion. It is called orbit equation since its solution is $r(\theta)$. The equation is inhomogeneous and second order in $\theta$. Certainly $f(r)$ or $f(1 / u)$ is theforce law of the orbit equation. 


\subsection{Evaluation of the magnitude of the velocity of the body at any point of its path}

From the velocity equation given by (2.3) we obtain the magnitude of the velocity as

$$
\begin{aligned}
& v^{2}=\dot{r}^{2}+r^{2} \dot{\theta}^{2}+r^{2} \dot{\beta}^{2}+r^{2} \dot{\mu}^{2}+r^{2} \dot{\alpha}^{2}+r^{2} \dot{\phi}^{2} \\
& v^{2}=\left(-h \frac{d u}{d \theta}\right)^{2}+r^{2}\left(\frac{h^{2}}{r^{4}}\right)+r^{2}\left(\frac{h_{1}^{2}}{r^{4}}\right)+r^{2}\left(\frac{h_{1}^{2}}{r^{4}}\right)+r^{2}\left(\frac{h_{2}^{2}}{r^{4}}\right)+r^{2}\left(\frac{h_{2}^{2}}{r^{4}}\right) \\
& v^{2}=h^{2}\left(\frac{d u}{d \theta}\right)^{2}+h^{2} u^{2}+2 h_{1}^{2} u^{2}+2 h_{2}^{2} u^{2} \\
& v^{2}=h^{2}\left\{u^{2}+\left(\frac{d u}{d \theta}\right)^{2}\right\}+2 h_{1}^{2} u^{2}+2 h_{2}^{2} u^{2}(2.38) \\
& v^{2}=h^{2}\left\{u^{2}+\left(\frac{d u}{d \theta}\right)^{2}\right\}+2 u^{2}\left(h_{1}^{2}+h_{2}^{2}\right)(2.39)
\end{aligned}
$$

The equation of an ellipse referred to its focus is given by

$$
\begin{aligned}
& r=\frac{l}{1+\varepsilon \cos \theta} ; \quad \frac{1}{r}=\frac{1+\varepsilon \cos \theta}{l} ; u=\frac{1}{r} \\
& u=\left(\frac{1}{l}+\frac{\varepsilon \cos \theta}{l}\right) ; \quad\left(\frac{d u}{d \theta}\right)^{2}=\left(\frac{-\varepsilon \sin \theta}{l}\right)^{2}(2.41) \\
& v^{2}=h^{2}\left\{\left(\frac{1+\varepsilon \cos \theta}{l}\right)^{2}+\left(\frac{-\varepsilon \sin \theta}{l}\right)^{2}\right\}+2\left(\frac{1+\varepsilon \cos \theta}{l}\right)^{2}\left(h_{1}^{2}+h_{2}^{2}\right)(2
\end{aligned}
$$

Since the tangential oscillation is very small we assume in this study that the constants $h_{1} \approx h_{2} \approx h$.

$$
\begin{aligned}
& v^{2}=h^{2}\left(\frac{1+2 \varepsilon \cos \theta+\varepsilon^{2} \cos ^{2} \theta+\varepsilon^{2} \sin ^{2} \theta}{l^{2}}\right)+4 h^{2}\left(\frac{1+\varepsilon \cos \theta}{l}\right)^{2} \\
& v^{2}=h^{2}\left(\frac{1+2 \varepsilon \cos \theta+\varepsilon^{2}}{l^{2}}\right)+4 h^{2}\left(\frac{(1+\varepsilon \cos \theta)^{2}}{l^{2}}\right)(2.44) \\
& v^{2}=h^{2}\left(\frac{1+2 \varepsilon \cos \theta+\varepsilon^{2}+1-1}{l^{2}}\right)+4 h^{2}\left(\frac{(1+\varepsilon \cos \theta)^{2}}{l^{2}}\right)(2.45) \\
& v^{2}=\frac{h^{2}}{l}\left(\left(\frac{2(1+\varepsilon \cos \theta)}{l}\right)-\frac{\left(1-\varepsilon^{2}\right)}{l}\right)+4 h^{2}\left(\frac{(1+\varepsilon \cos \theta)^{2}}{l^{2}}\right)(2.46) \\
& v^{2}=\frac{h^{2}}{l}\left(\frac{2}{r}-\frac{1}{a}\right)+\frac{4 h^{2}}{r^{2}}(2.47) \\
& v^{2}=\varphi\left(\frac{2}{r}-\frac{1}{a}+\frac{4 l}{r^{2}}\right)(2.48) \\
& v^{2}=\varphi\left(\frac{2}{r}-\frac{1}{a}+\frac{4(1+\varepsilon \cos \theta)}{r}\right)
\end{aligned}
$$

where $h^{2}=\varphi l(l$ is the semi-latus rectum of the ellipse), $a$ is the major semi-axis. Of course, for a fixed major semi-axis, $h$ determines the eccentricity. So the magnitude of the velocity needs to increase by a factor $\sqrt{4 l / r^{2}}$ in order to change the orbit as a result of the added tangential oscillating phase.

Thus equation (2.49) is the required equation which describes the magnitude of the velocity of the body at any point of its path as it translates and oscillates elliptically. The first two terms in the parenthesis of (2.49) is referred to as the critical speed or the critical velocity while the third term is the perturbation. The perturbation 
term is caused by the added effect of the tangential oscillating motion of the body. The magnitude of the path velocity of the body converges to the critical velocity in the absence of the tangential oscillating angles.

\section{Discussion of results}

The central force field is specified by four independent generalized coordinates. It has a tangential oscillating phase which is determined by the vertical oscillating angles. As the oscillating angles increases the force tends to be more negative and attractive. The first two terms in the parenthesis of (2.8) gives the exact radial force and the orbital angular force. The orbital angular force is acting perpendicularly to the direction of the radius. If, $\ddot{r}=\dot{r}=0$, then the path is a circle.

The differential orbit equation (DOE) given by (2.34) is second order and inhomogeneous in $u(\theta)$. Of course, the force field that is responsible for the orbit equation is negative and hence highly attractive. This is expected of a motion that is central in character. The DOE has a tangential oscillating phase. The oscillating phase is determined by the vertical oscillating angles. In case, $l=0$ then $\theta$ is a constant, and the path is a straight line through the origin, that is, the path is homogeneous.

The magnitude of the velocity of the body at any point of its path as shown (2.48) is quadratic in nature. Hence it would have a positive maximum and negative minimum value as a function of the radius. The positive value of $v$ corresponds to the velocity in the upper plane of the ellipse, while the negative value corresponds to the velocity in the lower plane of the ellipse. The first two terms in $v^{2}$ is referred to as the critical velocity while the last term is the perturbation. The magnitude of the velocity is strictly the value of the critical velocity as $r \rightarrow \infty$. Thus the magnitude of the velocity is maximum at the perihelion and minimum at the aphelion.

\section{Conclusion}

In general, we have in this study solved the problem of the motion of a body in a plane polar coordinate system which is subject to a central attractive force which is known and, in addition, a drag oscillating force which acts tangentially. The oscillating energy $E_{\text {osc }}$ which determines how energy is conveyed up and down in the oscillating phase is relatively determined by the vertical spin oscillating angles.

The result of the total energy is appreciably different from the motion in a central force field without a drag oscillating force. The new force law now comprises of the radial and the tangential oscillating parts which reduces the strength of the attractive central force. The knowledge of this type of central force motion which we have developed in this work can be extended from plane polar coordinate system to that of spherical and cylindrical polar coordinate systems.

\section{Appendix}

Let us consider the rotational motion of a body of mass $m$ about a fixed origin say, $O$, in an elliptical polar coordinate system. Suppose the body is also oscillating up and down about its equilibrium position as it translates rotationally round the fixed origin. The body thus possesses translational and rotational elliptical motion with polar coordinates $(r, \theta)$ and tangential spin oscillating motion described by the vertical displacement $C \rightarrow D \rightarrow C \rightarrow B \rightarrow C$ and repeatedly in the $y$-direction. The geometry of the analytical requirements is shown in fig. A. 1 .

The reader should take note that the oscillation of the body is not entirely out of the elliptical orbit of rotation. Rather the displacement $D$ and $B$ above and below $C$ is very small. The oscillation is still within the limits of the axis of rotation $C$. We have only decided to stretch $D$ and $B$ above and below $C$ considerably enough in order to reveal the geometrical concept required for the analytical calculation.

There are six possible degrees of freedom or generalized coordinates exhibited by the motion body under this circumstance: (i) translational and rotational in the elliptical plane $(r, \theta)$,(ii) the plane of upward oscillations $(\beta, \mu)$ and (iii) the plane of downward oscillations $(\alpha, \phi)$.

We shall compute separately the tangential spin oscillating motions in both oscillating frames and eventually combine the result with the orbital elliptical plane motion. In this study, we assume that the angular displacements in the tangential spin oscillating frames are not equal and so the system under study is not radially symmetric. Consequently, there is the existence of torque due to the non uniformity of the radii distances.

Accordingly, we can now develop relationships between the various areas indicated on fig. A. 1, with the goal to find the formula for the area swept out by the elliptical plane polar motion, and the result obtained from this is then added to the tangential oscillating triangle sections $D \hat{O} C$ and $C \hat{O B}$ respectively. 
From fig. (A. 1), $P$ and $Q$ are very small upward and downward displacements from the equilibrium axis of rotation $C$, that is, regions in the upper and lowertriangular swept segments of the upper and lower elliptical plane. Our first task would be to connect all these oscillating spin angular degrees of freedom into an expression in terms of $P$ and $Q$.

For clarity of purpose, let us define the various symbols which we may encounter in our calculations : (i) the elliptical radius $r$ (ii) the plane of upward oscillations $(\beta, \mu)$, that is subtended from the upper elliptical plane (iii) the plane of downward oscillations $(\alpha, \phi)$, that is subtended from the bottom or the lower part of the elliptical plane (iv) the elliptic orbital plane angle $\theta$ (v) the upper tangential oscillating spin angle $\mu$ (vi) the lower tangential oscillating spin angle $\phi$ (vii) the upper and lower orbital spin oscillating angles $\beta$ and $\alpha$.

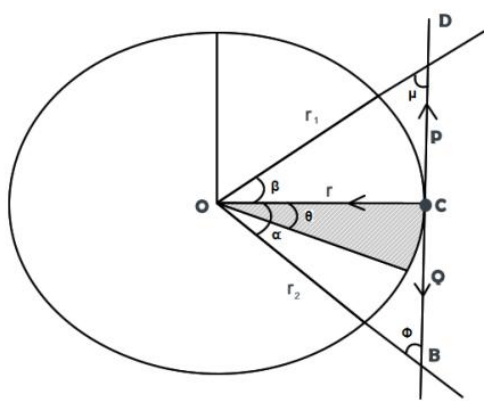

Fig. A.1. Represents the elliptical and oscillating motion of a body in a central-force field. The body is oscillating up and down about the axis of rotation $C$. Where $\triangle D \hat{O} C$ (frame I) and $\Delta C \hat{O} B$ (frame II) are the upper and lower projections of the tangential oscillating phases onto the plane of the ellipse, the lines $\overline{D C}(P)$ and $\overline{C B}(Q)$ are very small displacements from the axis $C$, we have only stretched them to make the geometry of the figure clear enough for the calculation. However, $\beta$ is the upper radial orbital oscillating angle and $\alpha$ is the lower radial orbital oscillating angle. Note that both of them are projections of the tangential oscillating plane onto the orbital elliptic plane.

In frame I : we obtain from $\Delta D \hat{O} C$

$r_{1}=r \operatorname{cosec} \mu \quad ; P=r_{1} \sin \beta=r \sin \beta \cos e c \mu$ (A. 1)

In frame II : we obtain from $\triangle C \hat{O} B$

$r_{2}=r \operatorname{cosec} \phi \quad ; \quad Q=r_{2} \sin \alpha=r \sin \alpha \operatorname{cosec} \phi$

In the orbital plane of rotational and translational motion, the position vector $\vec{r}$ of the body is given by $\vec{r}=x i=r \cos \theta i(\mathrm{~A} .3)$

However, the combination of the rotational and translational motion, with the vertical spin oscillating frames (acting in the $y$-direction), we have

$$
\begin{aligned}
& \vec{r}=x i+y j=x i+P j^{\uparrow}+Q j^{\downarrow}=r\left(\cos \theta i+\sin \beta \operatorname{cosec} \mu j^{\uparrow}+\sin \alpha \operatorname{cosec} \phi j^{\downarrow}\right)(\text { A.4) } \\
& \hat{r}=\frac{\partial \vec{r}}{\partial r}=\left(\cos \theta i+\sin \beta \operatorname{cosec} \mu j^{\uparrow}+\sin \alpha \operatorname{cosec} \phi j^{\downarrow}\right) \\
& \vec{r}=r \hat{r}=r \hat{r}(\theta, \beta, \mu, \alpha, \phi)(\text { A.6) } \\
& \hat{\theta}=\frac{\partial \hat{r}}{\partial \theta}=-\sin \theta i \quad ; \quad \frac{\partial \hat{\theta}}{\partial \theta}=-\cos \theta i=-\hat{r} \\
& \frac{\partial \hat{\beta}}{\partial \beta}=-\sin \beta \operatorname{cosec\mu } j^{\uparrow}=\frac{\cos \beta}{\cos \beta}\left(-\sin \beta \operatorname{cosec} \mu j^{\uparrow}\right) \\
& \frac{\partial \hat{\beta}}{\partial \beta}=\frac{\sin \beta}{\cos \beta}\left(-\cos \beta \operatorname{cosec} \mu j^{\uparrow}\right)=-\tan \beta \hat{\beta}
\end{aligned}
$$


$\hat{\mu}=\frac{\partial \hat{r}}{\partial \mu}=-\sin \beta \cos \mu \operatorname{cosec}{ }^{2} \mu j^{\uparrow}$

$\frac{\partial \hat{\mu}}{\partial \mu}=\sin \beta \sin \mu \operatorname{cosec} c^{2} \mu j^{\uparrow}+2 \sin \beta \cos \mu \cot \mu \operatorname{cosec}{ }^{2} \mu j^{\uparrow}$

$\frac{\partial \hat{\mu}}{\partial \mu}=\frac{\cos \mu}{\cos \mu}\left(\sin \beta \sin \mu \operatorname{cosec} c^{2} \mu j^{\uparrow}\right)+2 \cot \mu\left(\sin \beta \cos \mu \operatorname{cosec}{ }^{2} \mu j^{\uparrow}\right)$

$\frac{\partial \hat{\mu}}{\partial \mu}=\frac{\sin \mu}{\cos \mu}\left(\sin \beta \cos \mu \operatorname{cosec}{ }^{2} \mu j^{\uparrow}\right)+2 \cot \mu\left(\sin \beta \cos \mu \operatorname{cosec}^{2} \mu j^{\uparrow}\right)$

$\frac{\partial \hat{\mu}}{\partial \mu}=-\tan \mu \hat{\mu}-2 \cot \mu \hat{\mu}$ (A.14)

$\hat{\alpha}=\frac{\partial \hat{r}}{\partial \alpha}=\cos \alpha \operatorname{cosec} \phi j^{\downarrow} ; \frac{\partial \hat{\alpha}}{\partial \alpha}=-\sin \alpha \operatorname{cosec} \phi j^{\downarrow}=\frac{\cos \alpha}{\cos \alpha}\left(-\sin \alpha \operatorname{cosec} \phi j^{\downarrow}\right)$

$\frac{\partial \hat{\alpha}}{\partial \alpha}=\frac{\sin \alpha}{\cos \alpha}\left(-\cos \alpha \operatorname{cosec} \phi j^{\downarrow}\right)=-\tan \alpha \hat{\alpha}$

$\hat{\phi}=\frac{\partial \hat{r}}{\partial \phi}=-\sin \alpha \cos \phi \operatorname{cosec}{ }^{2} \phi j^{\downarrow}$ (A.17)

$\frac{\partial \hat{\phi}}{\partial \phi}=\sin \alpha \sin \phi \operatorname{cosec} c^{2} \phi j^{\downarrow}+2 \sin \alpha \cos \phi \cot \phi \operatorname{cosec}{ }^{2} \phi j^{\downarrow}$

$\frac{\partial \hat{\phi}}{\partial \phi}=\frac{\cos \phi}{\cos \phi}\left(\sin \alpha \sin \phi \operatorname{cosec}{ }^{2} \phi j^{\downarrow}\right)+2 \cot \phi\left(\sin \alpha \cos \phi \operatorname{cosec}{ }^{2} \phi j^{\downarrow}\right)($ A.19)

$\frac{\partial \hat{\phi}}{\partial \phi}=\frac{\sin \phi}{\cos \phi}\left(\sin \alpha \cos \phi \operatorname{cosec}{ }^{2} \phi j^{\downarrow}\right)+2 \cot \phi\left(\sin \alpha \cos \phi \operatorname{cosec}^{2} \phi j^{\downarrow}\right)($ A. 20$)$

$\frac{\partial \hat{\phi}}{\partial \phi}=-\tan \phi \hat{\phi}-2 \cot \phi \hat{\phi}($ A.21)

Also we know from the rule of differentiation that

$m \frac{d}{d t}\left(r^{2}\right)=m \frac{d}{d t}(r . r)=m\left(\frac{d r}{d t} r+r \frac{d r}{d t}\right)=m\left(2 r \frac{d r}{d t}\right)=m\left(2 \frac{d r^{2}}{d t}\right)=\frac{d}{d t}\left(\frac{1}{2} m r^{2}\right)$

Hence, in order to remove the factor of 2 which appears in (A. 22), we then introduce the factor of half.

\section{References}

[1]. Whittaker E. T. (1937). “Analytical Dynamics”. Cambridge, England : Cambridge University Press.

[2]. Meriam J. L. and Kraige.L. G. (2009).Engineering mechanics, DYNAMICS, $5^{\text {th }}$ Edition.

[3]. Rosu L. and Haret C. (1999). "Classical Mechanics". Physics Education. [arxiv.org:physics/9909035]

[4]. Keith R. Symon. (1979). "Mechanics". 3rd edition. Addison-Wesley publishing company.

[5]. Weisstein Eric W. (2007). "Central Force”.(http://science world.wolfram.com/physics/CentralForce.html). Science World. 